\title{
Alterations of Signaling Pathways in Essential Thrombocythemia with Calreticulin Mutation
}

\author{
Wuhan Hui \\ Wei Zhang \\ Congyan Liu \\ Suigui Wan \\ Wanling Sun \\ Li Su
}

Department of Hematology, Xuan Wu Hospital, Capital Medical University, Beijing, People's Republic of China
Correspondence: Li Su

Department of Hematology, Xuan Wu Hospital, Capital Medical University,

Beijing, People's Republic of China

Tel/Fax +86-10-83198476

Email suli@xwhosp.org
Purpose: Though mutations of the calreticulin $(C A L R)$ gene have been identified in essential thrombocythemia patients, the detailed mechanisms for CALR mutations have not been completely clarified. Our study is aimed at characterizing alteration of protein expression in ET patients with mutated $C A L R^{\text {del52 }}$ and further recognizing possible involvement of signaling pathways associated with $C A L R$ mutations.

Patients and Methods: Protein pathway array was performed to analyze the expression levels of proteins involved in various signaling pathways in peripheral blood neutrophils from $18 \mathrm{ET}$ patients with mutated $C A L R^{\text {del52 }}, 20 \mathrm{ET}$ patients with $J A K 2^{V 617 F}$ mutation and 20 controls.

Results: We found 20 proteins differentially expressed in ET patients with mutated $C A L R^{\text {del52 }}$ compared with healthy controls. These proteins were associated with molecular mechanisms of cancer in ingenuity pathways analysis (IPA) network. We identified top ten canonical pathways which including apoptotic pathways and cellular cytokine pathways might participate in pathogenesis of ET with mutated $C A L R^{\text {del52 }}$. Additionally, there were 8 proteins found to be dysregulated differently between ET patients with mutated $C A L R^{\text {del52 }}$ and those with $J A K 2^{V 617 F}$ mutation. These proteins might be related to the unique signaling pathways activated by $C A L R^{\text {del52 }}$ mutation which were different to JAK/STATs pathway by $J A K 2^{V 617 F}$ mutation.

Conclusion: Our study demonstrated that numerous alterations of signaling proteins and pathways in ET patients with mutated $C A L R^{d e l 52}$. These findings could help to gain insights into the pathological mechanisms of ET.

Keywords: essential thrombocytosis, $C A L R^{\text {del52 }}$ mutation, protein pathway array, signaling proteins

\section{Introduction}

As a subcategory of myeloproliferative neoplasms (MPNs), essential thrombocytosis (ET) is characterized by an elevated platelets number, enhanced megakaryocyte count in bone marrow and increased risk for vascular events such as thrombosis or bleeding. ${ }^{1}$ More than $85 \%$ of patients with ET carry driver gene mutations, including mutation of janus kinase 2 gene $\left(J A K 2^{V 617 F}\right)$, thrombopoietin receptor $(M P L)$ genes and calreticulin $(C A L R)$ genes. $^{2-8}$ It's reported that JAK2V617F is mutated in nearly $50-60 \%$ of ET, ${ }^{2-4}$ and MPL mutation is detected in $5 \%$ of cases. ${ }^{5,6}$ The $J A K 2^{V 617 F}$ mutation causes constitutive, independent activation of downstream JAK/STAT signaling and eventually disruption of gene transcription. ${ }^{2-4}$ Furthermore, JAK2 $2^{V 617 F}$ mutation alone has been shown to result in MPN phenotypes in vivo and in vitro models. ${ }^{9,10}$ In 2013 , mutated CALR genes 
were found in $50 \%$ of $J A K 2 / M P L$ negative ET. ${ }^{7,8}$ Recent datas have shown that mutated CALR directly binds MPL and activates its downstream JAK/STAT signaling. ${ }^{11-13}$ However, the detailed mechanisms for $\operatorname{CALR}\left(C A L R^{\text {del52 }}\right)$ mutation has not been fully unraveled.

In this study, we undertook a comprehensive screening of signaling proteins using Protein Pathway Array (PPA) ${ }^{14}$ for ET patients with $C A L R^{\text {del52 }}$ mutation to characterize the dysregulation of signaling protein expression and explored the potential involvement of signaling pathways related to $C A L R^{\text {del52 }}$ mutation. PPA is a proteomic method of analyzing alterations in intracellular protein expression in multiple signaling pathways, including apoptosis, cell proliferation and differentiation, invasion, cell cycle regulation, DNA repair and angiogenesis. ${ }^{14-16}$ Using this method, several signaling proteins differentially expressed between $C A L R^{\text {dels2 }}$ and $J A K 2^{V 617 F}$ mutated ET and healthy controls were identified.

\section{Materials and Methods}

\section{Patients and Samples}

Peripheral blood samples were collected from patients with newly diagnosed ET patients at Xuan Wu Hospital, Capital Medical University January 2010 and April 2011. Granulocytes were isolated using the method previously reported. ${ }^{17}$ Eighteen ET patients with mutated $C A L R^{\text {del52 }}$ were included in this study. In addition, $20 \mathrm{JAK2} 2^{\text {V617F }}$ mutation ET patients were included for comparison. The diagnosis of ET was established according to 2008 revision of WHO classification of myeloid neoplasms and acute leukemia. ${ }^{18}$ Control samples $(n=20)$ were collected from sex and age matched healthy volunteers. The study was approved by the ethics committee of Xuan Wu Hospital. All participants signed an informed consent form.

\section{Protein Pathway Array}

Proteins were extracted from neutrophils of each sample and then $300 \mu \mathrm{g}$ extracted protein was loaded in one well across the entire width of the gel. Furthermore, the separated proteins were electrophoretically transferred to a nitrocellulose membrane (Bio-Rad, Hercules, USA), which was then blocked using blocking buffer ( $3 \%$ BSA in $1 \times$ TBST containing $20 \mathrm{mM}$ Tris-HCL, $100 \mathrm{Mm} \mathrm{NaCL}$ and $0.1 \%$ Tween-20) at room temperature for $1 \mathrm{~h}$. Next, the membrane was clamped with a Western blotting manifold in order to isolate 20 channels across the membrane (Mini-PROTEAN II Multiscreen apparatus, Bio-Rad Laboratories, Inc.). Three or two antibodies were added to each channel and the first set of 35 antibodies were allowed to hybridize proteins at $4^{\circ} \mathrm{C}$ overnight as described previously. ${ }^{15}$ The blot was hybridized for $45 \mathrm{~min}$ with horseradish peroxidase conjugated secondary antibodies at room temperature. Then the membranes were developed with chemiluminescence substrate, and signals were caught by the ChemiDoc XRS system (Bio-Rad Laboratories, Inc.). The same membrane was stripped off and then used to detect a second set of 33 proteins-specific antibodies as described above. Total 128 antibodies were used for each membrane (Table 1). Proteins quantification was analyzed using densitometric scanning (Quantity One software; Bio-Rad Laboratories, Inc.) and normalized by internal standards.

\section{Signaling Pathway and Network Analysis}

Ingenuity Pathway Analysis (IPA) (http://www.ingenuity. com) was used for functional pathway and network analysis. ${ }^{14}$ Differentially expressed proteins identified by PPA were input into the IPA software and mapped to their corresponding gene objects in the Ingenuity Knowledge Base. In canonical pathways analyses, IPA was performed to determine which functional pathways were significantly related to

Table I List of Antibodies Included in the Protein Pathway Array

Cell signaling: Akt, Axin, $\beta$-catenin, Calretinin, $\mathrm{CPKC} \alpha$, ErkI/2,

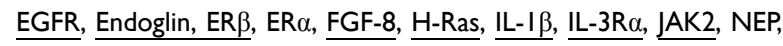
PTEN, Rab 7, Raf-B, Rap I, SOCSI, SOCS3, Wnt-I, CREB, Flt-3/Flk-2, Her2, Hsp90, IGFBP5, JaggedI, K-Ras, KAII, p38 $\alpha / \beta$, Maspin, nm23$\mathrm{HI} / 2 / 3$, PERK, patched

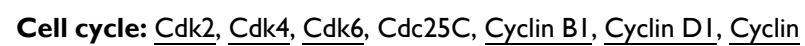
E, Cdc42, p27, 14-3-3B, Cdc2p34, Cdc25B, CHKI, MDM2, Aurora A/ AIK

Transcription factor: Stat3, Stat5, elF4B, WTI, PDEF, Pax-2, ASCLI, FKHR, HDACI, SLUG, SRC-I, HESI, p63, TTF-I, Nothc4, E2F-I, Stat I, NFATcI, TCF-I, E2A, ATF-I, Nkx-3.I, HMG-I,

Apoptosis/Autophagy: Bax, BID, Bak, NFkB p50, Bcl-6, Bcl-xL, RIP,

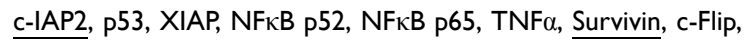
BECNI, Bad, Bcl-2

Angionesis: $\underline{\mathrm{EPO}}, \underline{\mathrm{HIF}-\mathrm{I} \alpha}, \underline{\mathrm{HIF}-2 \alpha}, \underline{\mathrm{HIF}-3 \alpha}, \underline{\mathrm{TGF}-\beta}, \mathrm{VEGF}$

DNA repair: TDPI, TFIIH P89, ERCCI, PCNA

Epithelial-to-mesenchymal transition/Adhesion: E-cadherin, N-cadherin, Mesothelin, PSM, L-Selectin, Ep-CAM, ICAM-I, VCAM-I, Vimentin, OPN, E-Selectin, HCAM, CD33

Others: Cytokeratin 5, Cytokeratin 18, FAH, FT $\alpha$, uPA, Factor XIII B, uPAR, PSCA, Cytokeratin 19, Keratin 10, Glutamine Synthetase, DRGI, Eg5

Notes: Underlines indicate detectable expression in samples from ET patients or healthy controls. 
the input gene set through a Fisher's exact test. Furthermore, IPA identified local networks that were remarkably enriched for the input genes by computational algorithms.

\section{Statistical Analysis}

Significant Analysis of Microarray (SAM) tool (http:// www-stat.stanford.edu/ tibs/SAM/) was adopted to identify the proteins with differential expression between different groups. Fisher's Exact Test and Student $t$-Test were applied to compare laboratory findings and categorical variables. Statistical analyses were conducted with the software SPSS version 17.0 (SPSS Inc, Chicago, IL, USA) software. The $q$-value and $p$-value of less than 0.05 were considered as statistically significant.

\section{Results}

\section{Patient-s' Clinicohematological Characteristics}

The clinicopathological characteristics of ET patients are summarized in Table 2. The median age of patients with $C A L R^{\text {del52 }}$ and $J A K 2^{V 617 F}$ mutation was $48.4 \pm 14.1$ years (range, 25 and 74), and 58.4 \pm 12.9 years (range, 34-78), respectively. $C A L R^{\text {del52 }}$ mutated patients were younger than JAK2 ${ }^{\text {V617F }}$ mutated ones $(\mathrm{P}=0.029)$, consistent with previous reports. ${ }^{19}$ Compared with the JAK2 $2^{V 617 F}$ mutated group (40\%), $C A L R^{\text {del52 }}$ mutation was found more frequent in males (56\%). Patients with a $C A L R^{\text {del52 }}$ mutation had lower white blood cell count (median of $7.1 \times 10^{9} / \mathrm{L}$ versus $10.1 \times 10^{9} / \mathrm{L}$; $\mathrm{P}=0.007$ ), higher platelet counts (median of $883 \times 10^{9} / \mathrm{L}$ versus
$694 \times 10^{9} / \mathrm{L} ; \mathrm{P}=0.02$ ), and appeared to have more frequent thrombocytosis above $1000 \times 10^{9} / \mathrm{L}(44 \%$ vs $20 \%)(P$ $=0.164)$. Median hemoglobin value was $131 \mathrm{~g} / \mathrm{L}$ and did not differ from JAK2 ${ }^{\mathrm{V} 617 F}$ mutated group $(P=0.073)$ (Table 2).

\section{Identification of Differentially Expressed Proteins in ET with CALR ${ }^{\text {del}} 52$ Mutation}

Of 128 proteins examined, 71 proteins were detected in all 38 samples. We found 20 proteins dysregulated in ET patients with $C A L R^{\text {del52 }}$ mutation compared with those in healthy controls $(\mathrm{q}<0.1)$. Among them, 13 proteins including PTEN, Erk1/2, Rap1, Cdc42, Raf-B, Axin, eIF4B, c-IAP2, NFkB p50 Bcl-X1, CyclinD1, TGF- $\beta$, and SRC-1 were overexpressed, while 7 proteins including TDP1, ER $\beta$, E-cadherin, $\mathrm{cPKC} \alpha, \mathrm{Cdc} 2$, Survivin and p27 were downregulated. These proteins were associated with molecular mechanisms of cancer in IPA network (Figure 1).

\section{Signaling Pathways Altered in ET with CALR ${ }^{\text {del52 }}$ Mutation}

To determine the functional pathways most affected by differentially expressed proteins, these 20 proteins were uploaded into IPA for functional annotation and pathway analysis. We identified top 10 canonical pathways which included Cyclins and cell cycle regulation, IL-12 signaling and production in Macrophages, HGF signaling, Protein kinase A signaling, ILK signaling, Regulation of the epithelial-mesenchymal transition pathway, PI3K-AKT signaling, IL-8 signaling, Apoptosis signaling and PTEN signaling (Figure 2).

Table 2 Main Features of Patients with ET

\begin{tabular}{|c|c|c|}
\hline & No. of Patients with CALR ${ }^{\text {del52 }}(n=\mid 8)$ & No. of Patients with JAK2V6I7F $(n=20)$ \\
\hline Gender;n(\%) & & \\
\hline Male & $10(56)$ & $8(40)$ \\
\hline Female & $8(44)$ & $12(60)$ \\
\hline Age in years; medina (range) & $5 I(25-74)$ & $60(33-78)$ \\
\hline WBC, $\times 10^{9} / \mathrm{L} ;$ median(range) & $7.1(5.1-12.4)$ & $10.1(5.78-15.68)$ \\
\hline$>1 \mid \times 10^{9} / \mathrm{L} ; \mathrm{n}(\%)$ & $2(11)$ & $7(35)$ \\
\hline Hemoglobin, g/L;median(range) & $13 \mid(\mid 17-164)$ & $140(122-154)$ \\
\hline Platelet, $\times 10^{9} / \mathrm{L} ;$ median(range) & $883(560-1793)$ & $694(46 I-97 I)$ \\
\hline$>1000 \times 10^{9} / \mathrm{L} ; \mathrm{n}(\%)$ & $8(44)$ & $4(20)$ \\
\hline Splenomegaly at diagnosis;n(\%) & $5(28)$ & $7(35)$ \\
\hline Vascular risk factors; $\mathrm{n}(\%)^{*}$ & $4(22)$ & $7(35)$ \\
\hline History of thrombosis; $n(\%)$ & $3(17)$ & $7(35)$ \\
\hline
\end{tabular}

Notes: *Vascular risk factors include Hypertension, Diabetes mellitus, and Hyperlipidemia. 


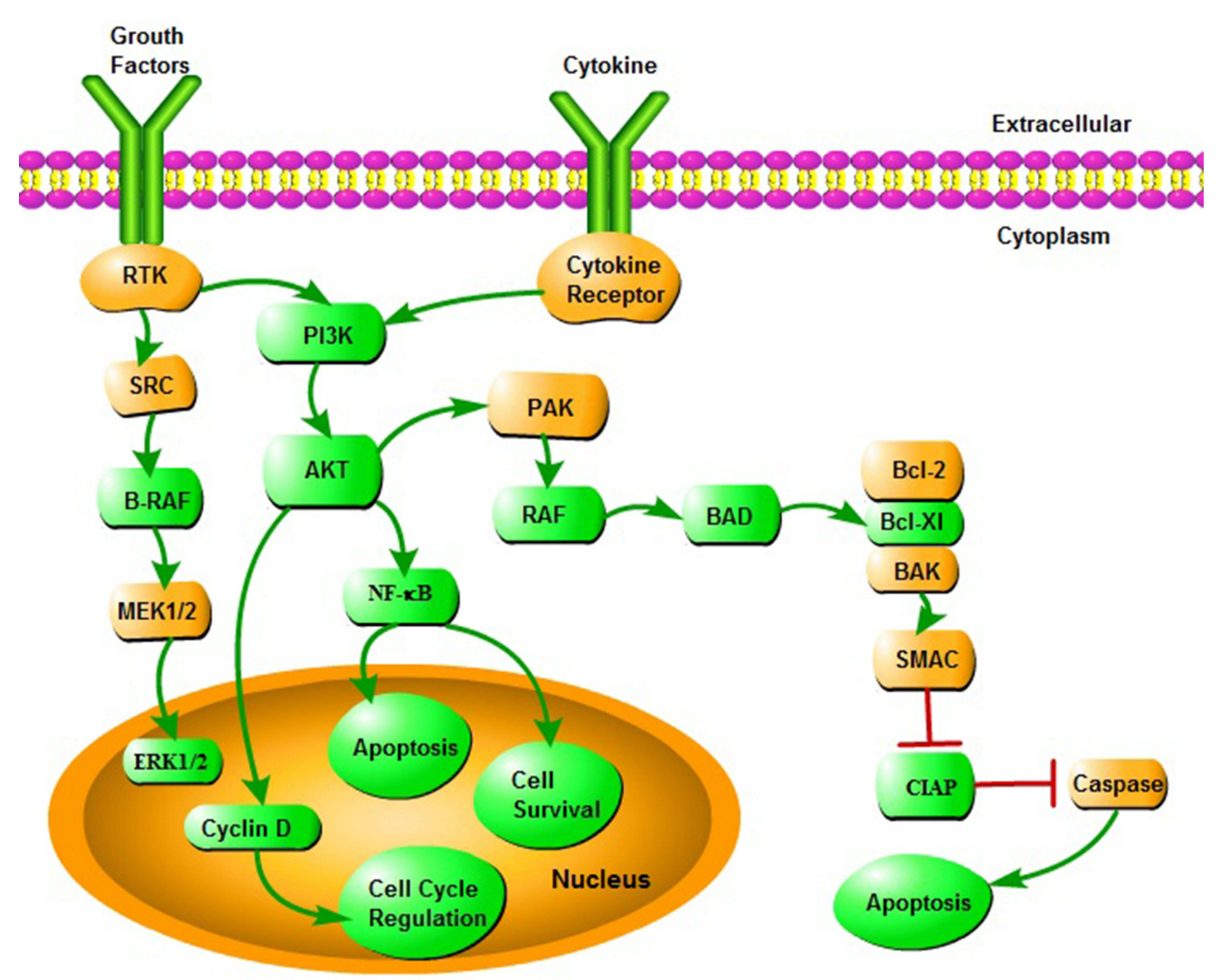

Figure I The dysregulated proteins from the ET with CALR mutation highlighted in green within the current signaling pathway by IPA analysis.

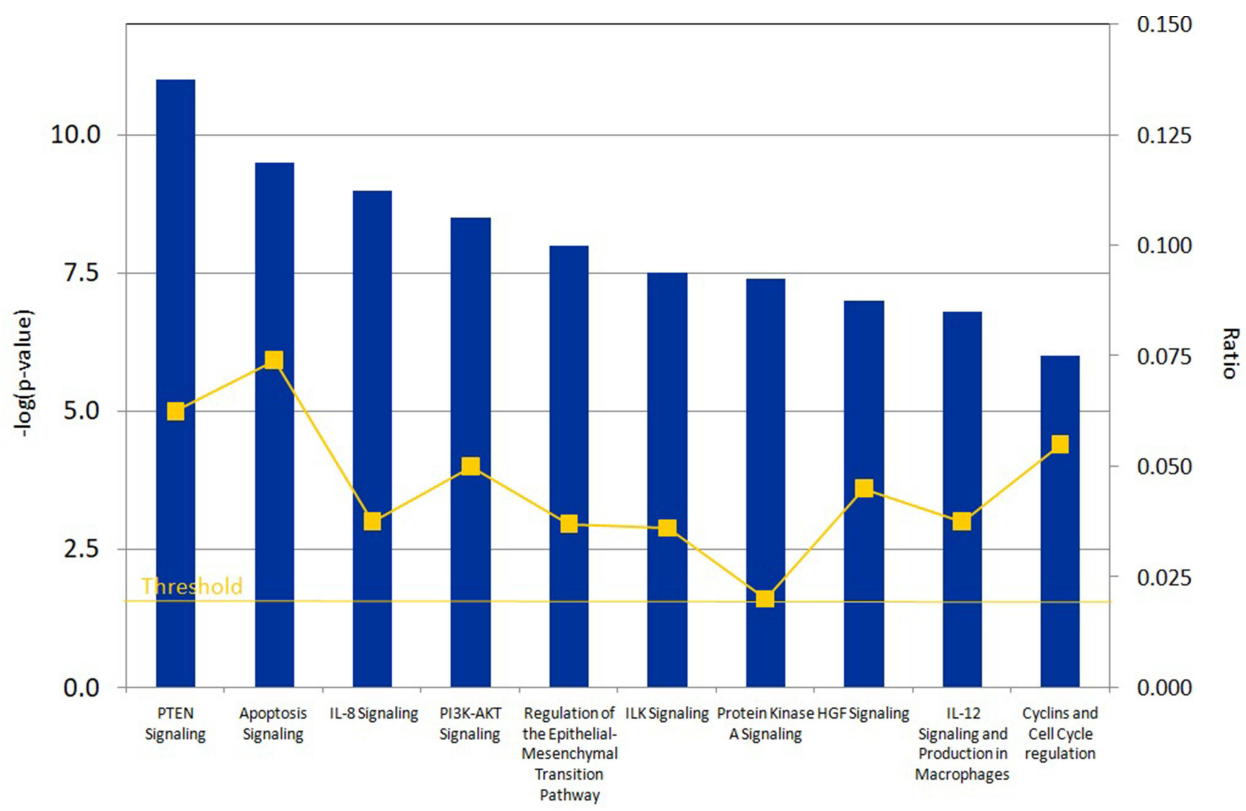

Figure 2 The top canonical pathways affected by 20 dysregulated proteins in ET with CALR mutations.

\section{Signaling Network in ET with CALR ${ }^{\text {del52 }}$ Mutation}

To further investigate the protein-protein interaction network in addition to the major regulators, IPA was conducted on the 20 proteins (genes) identified by PPA, and then the signal network was built (Figure 3). This complex network in $C A L R^{\text {del52 }}$ mutation appeared to affect many cellular functions, including differentiation of cells 


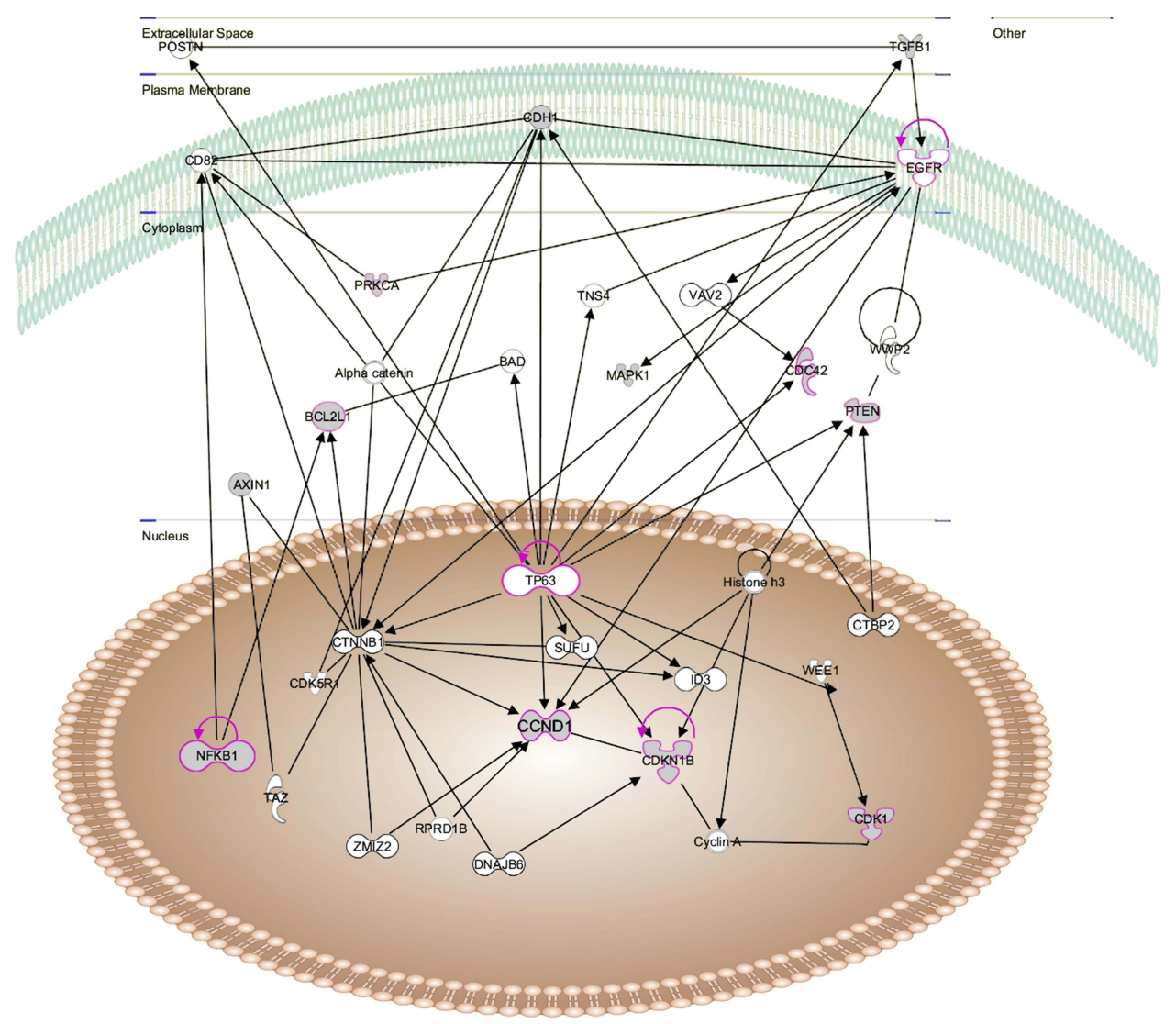

Figure 3 Protein signaling network altered in ET with CALR mutations as determined by IPA. The dysregulated proteins shaded in gray. The network was shown graphically as nodes(proteins) and edges (the biological association between the nodes). The non-colored nodes were not evaluated in this study but identified by IPA as significant nodes involved in the network. The various shaped nodes represent the functional class of the proteins.

and cell cycle progression. Chronic inflammatory disorders were influenced as well. In this network analysis, core regulators included E-cadherin $(C D H 1), \operatorname{PTEN}(P T E N)$, CyclinD1 (CCND1), NFאB (NFKB1), p27 (CDKN1B).

\section{Comparative Analysis of Protein Expression in ET with CALR ${ }^{\text {del52 }}$ or JAK2 ${ }^{\text {V6/7F }}$ Mutation}

In order to determine the various changes in activated signaling pathways between ET patients with $C A L R^{\text {del52 }}$ mutation and those with $J A K 2^{V 617 F}$ mutation, the protein expression levels in these 2 groups were compared and 8 proteins (CyclinD1, Stat5, HIF1 $\alpha$, IL-1 $\beta$, TGF- $\beta$, p27, Erk1/2, ASCL) were found at different levels $(\mathrm{q}<0.05)$. These proteins might relate to the signaling pathways of activation by $C A L R^{\text {del52 }}$ mutation, which were different from JAK/STAT signaling by $J A K 2^{V 617 F}$ mutation.

\section{Discussion}

CALR mutations trigger JAK/STATs signaling dysregulation in ET, which is similar to $J A K 2$ V617F mutation, however, $C A L R$ mutations were reported to be associated with distinct disease features. ${ }^{19}$ We have also found that $C A L R^{\text {del52 }}$ mutation status showed a tendency towards younger age and higher platelet counts. It suggests that additional signaling pathways might determine the disease phenotype, and participate in the development and propagation of the disease. Investigation of signaling pathways is of significance for further understanding the pathogenesis of ET with CALR mutations as well as for discovering effective therapeutic targets.

Having used large scale proteomic analysis, we succeeded in identifying numerous signaling proteins that were altered in ET with $C A L R^{\text {del52 }}$ mutation. These 20 proteins involved cell signaling (Erk1/2, PTEN, Raf-B, Rap1, Axin, ER $\beta$, TGF- $\beta$ ), cell cycle (Cdc42, Cdc2, CyclinD1, p27), apoptosis (Bcl-X1, c-IAP2, NFאB p50, cPKC $\alpha$, Survivin), transcription factor (eIF4B, SRC-1), DNA repair (TDP1) and adhesion 
(E-cadherin). Among these proteins, a significant overexpression was identified for anti-apoptotic proteins (Bcl-Xl, c-IAP2), which might be associated with over-activation of signaling pathways against apoptosis.

Supporting this, the results of IPA analysis for these 20 differentially expressed proteins revealed that apoptosis signaling displayed a significant relationship with $C A L R^{\text {del52 }}$ mutated ET (Figure 2). It has been demonstrated that ET displayed dysregulation of apoptotic pathway. ${ }^{20,21}$ Thus, the up-regulated anti-apoptotic proteins discovered in the current study could be involved in apoptotic defects and pathogenesis in $C A L R^{\text {del52 }}$ mutated ET.

Emerging evidence has shown that cytokine production was associated with clinical symptoms and contributed to BM fibrosis and disease progression and adverse prognosis in MPN. ${ }^{22-24}$ Pourcelot et al showed that elevated levels of inflammatory cytokines such as IFN $\gamma$, IL8, IL4, VEGF, GM-CSF, PDGF, and MCP-1, occurred in ET. ${ }^{25}$ In line with these reported results, the current study discovered two significant pathways associated with cellular cytokine pathways (IL-8 signaling, and IL-12 signaling pathways), suggesting a potential role of inflammatory process in the disease progression of ET with $C A L R^{\text {del52 }}$ mutation.

One of the other top pathways discovered in this study was ILK signaling. ILK has been shown to be involved in $\alpha$ granule secretion of platelets and plays a main role in the regulation of platelet function. The ILK-deficient mice showed an increased bleeding time, and an obvious reduce in the stability of thrombi formed. ${ }^{26,27}$ Further work will be required to explore whether ILK signaling plays a role in thrombosis of ET with $C A L R^{\text {dels2 }}$ mutation. In addition, we discovered multiple other important signaling pathways which were reported to be involved in carcinogenesis and tumor progression, for example, PTEN signaling, HGF signaling, Regulation of the epithelial-mesenchymal transition pathway, cell cycle regulation (Figure 2). These signaling pathways might play a significant role in the pathogenesis and/or progression of ET with $C A L R^{\text {del52 }}$ mutation.

Furthermore, it is crucial to point out that the proteins identified in this study did not function independently but as a part of a complex signaling network. Therefore, signal networks were further built using IPA in our study to determine the interactions among these proteins (Figure 3). Out of these complicated networks, several core regulators in $C A L R^{\text {del52 }}$ mutated ET were identified. The finding of these core regulators with a higher degree demonstrated they had additional interactions with other molecules in the signaling network. For example, NF-kB
(NFKB1) was not only upregulated in $C A L R^{\text {del52 }}$ mutated ET compared with control cells, but also served as the central node to establish the interaction network (Figure 3), which suggested this transcription factor played a central role in $C A L R^{\text {del52 }}$ mutation. Recently, Kleppe et $\mathrm{al}^{28}$ reported that NF-kB signaling as a core pathway was constitutively active in MPN mouse models and NF$\mathrm{kB}$ was a key regulator of inflammation involved in disease process. Taken together, these results underscore a role of NF-kB signaling in MPN pathogenesis, and NF$\mathrm{kB}$ could be a potential therapeutic target for MPN.

Notably, in comparison of activated signaling pathways between $C A L R^{\text {del52 }}$ mutated and $J A K 2^{\text {V617F }}$ mutated ET patients, 8 proteins were found to be differentially expressed which included CyclinD1, Stat5, Erk1/2, IL-1 $\beta$, HIF1 $\alpha$, TGF$\beta$, p27, ASCL. Increased phosphorylated STAT3 and STAT5 expression has been previously described in JAK2-mutated MPNs, ${ }^{3,4,29}$ but STAT5 signaling patterns appeared to be distinct in CALR-mutated ET. MARIMO, a CALR-mutated cell line has been reported to possess markedly reduced levels of STAT5, pSTAT5, and constitutive activation of ERK1/2. ${ }^{30,31}$ Furthermore, Lau et $\mathrm{al}^{32}$ recently reported that STAT3 and STAT5 targeted genes were overexpression in granulocytes from ET patients with $J A K 2$ mutations but not from those with CALR mutations. Consistent with these data, we also observed decreased expression of STAT5 and increased expression of Erk1/2 in $C A L R^{\text {dels2 }}$-mutated compared with in $J A K 2^{V 617 F_{-}}$ mutated ET.

\section{Conclusion}

In conclusion, our study reveals that a broad dysregulation of signaling proteins and distinct signaling patterns in ET patients with $C A L R^{\text {del52 }}$ mutations, including apoptotic proteins and pathways, which suggest ET with $C A L R^{\text {del52 }}$ mutation might possess unique signaling pathways involving disease pathogenesis and progression. Our findings are of importance in gaining insights into the pathological mechanisms of ET. Future investigations will be more focused on exploring the roles of these proteins in affecting tumor behavior, and hopefully unravel new therapeutic targets.

\section{Acknowledgments}

We thank Dr. David Y. Zhang and Dr. Fei Ye (Mount Sinai Hospital) for the technical support in PPA experiments.

\section{Disclosure}

Dr Wuhan Hui reports grants from National Natural Science Foundation of China grants (grant nos. 
81400094), during the conduct of the study. The authors report no conflicts of interest in this work. The partial abstract of this paper was presented at the ASH Conference name "Alterations of Signaling Pathways in Essential Thrombocythemia with Calreticulin Mutation" as a poster presentation. The poster's abstract was published in "Poster Abstracts" in Blood Journal: https://doi. org/10.1182/blood.V128.22.4286.4286

\section{References}

1. Tefferi A, Pardanani A, Solomon CG. Essential thrombocythemia. $N$ Engl $J$ Med. 2019;381(22):2135-2144. doi:10.1056/ NEJMcp 1816082

2. James C, Ugo V, Le Couedic J-P, et al. A unique clonal JAK2 mutation leading to constitutive signalling causes polycythaemia vera. Nature. 2005;434(7037):1144-1148. doi:10.1038/nature03546

3. Kralovics R, Passamonti F, Buser AS, et al. A gain-of-function mutation of JAK2 in myeloproliferative disorders. $N$ Engl $J$ Med. 2005;352(17):1779-1790. doi:10.1056/NEJMoa051113

4. Kralovics R, Teo -S-S, Buser AS, et al. Altered gene expression in myeloproliferative disorders correlates with activation of signaling by the V617F mutation of Jak2. Blood. 2005;106(10):3374-3376. doi:10.1182/blood-2005-05-1889

5. Pikman Y, Lee BH, Mercher T, et al. MPLW515L is a novel somatic activating mutation in myelofibrosis with myeloid metaplasia. PLoS Med. 2006;3(7):e270. doi:10.1371/journal.pmed.0030270

6. Pardanani AD, Levine RL, Lasho T, et al. MPL515 mutations in myeloproliferative and other myeloid disorders: a study of 1182 patients. Blood. 2006;108(10):3472-3476. doi:10.1182/blood-200604-018879

7. Klampfl T, Gisslinger H, Harutyunyan AS, et al. Somatic mutations of calreticulin in myeloproliferative neoplasms. $N$ Engl $J$ Med. 2013;369(25):2379-2390. doi:10.1056/NEJMoa1311347

8. Nangalia J, Massie CE, Baxter EJ, et al. Somatic CALR mutations in myeloproliferative neoplasms with nonmutated JAK2. $N$ Engl J Med. 2013;369(25):2391-2405. doi:10.1056/NEJMoa1312542

9. Wernig G, Mercher T, Okabe R, et al. Expression of Jak2V617F causes a polycythemia vera-like disease with associated myelofibrosis in a murine bone marrow transplant model. Blood. 2006;107 (11):4274-4281. doi:10.1182/blood-2005-12-4824

10. Akada H, Yan D, Zou H, et al. Conditional expression of heterozygous or homozygous Jak2 $\mathrm{V} 617 \mathrm{~F}$ from its endogenous promoter induces a polycythemia vera-like disease. Blood. 2010;115 (17):3589-3597. doi:10.1182/blood-2009-04-215848

11. Elf S, Abdelfattah NS, Chen E, et al. Mutant calreticulin requires both its mutant $\mathrm{C}$-terminus and the thrombopoietin receptor for oncogenic transformation. Cancer Discov. 2016;6(4):368-381. doi:10.1158/2159-8290.CD-15-1434

12. Li J, Prins D, Park HJ, et al. Mutant calreticulin knockin mice develop thrombocytosis and myelofibrosis without a stem cell self-renewal advantage. Blood. 2018;131(6):649-661. doi:10.1182/ blood-2017-09-806356

13. Marty C, Pecquet C, Nivarthi H, et al. Calreticulin mutants in mice induce an MPL-dependent thrombocytosis with frequent progression to myelofibrosis. Blood. 2016;127(10):1317-1324. doi:10.1182/ blood-2015-11-679571

14. Zhang DY, Ye F, Gao L, et al. Proteomics, pathway array and signaling network-based medicine in cancer. Cell Div. 2009;4(1):20. doi:10.1186/1747-1028-4-20

15. Ye F, Che Y, McMillen E, et al. The effect of Scutellaria Baicalensis on the signaling network in hepatocellular carcinoma cells. Nutr Cancer. 2009;61(4):530-537. doi:10.1080/01635580902803719
16. Wang D, Ye F, Sun Y, et al. Protein signatures for classification and prognosis of gastric cancer a signaling pathway-based approach. Am J Pathol. 2011;179(4):1657-1666. doi:10.1016/j.ajpath.2011.06.010

17. Bauer S, Abdgawad M, Gunnarsson L, et al. Proteinase 3 and CD177 are expressed on the plasma membrane of the same subset of neutrophils. J Leukoc Biol. 2007;81(2):458-464. doi:10.1189/jlb.0806514

18. Vardiman JW, Thiele J, Arber DA, et al. The 2008 revision of the World Health Organization (WHO) classification of myeloid neoplasms and acute leukemia: rationale and important changes. Blood. 2009;114(5):937-951. doi:10.1182/blood-2009-03-209262

19. Al Assaf C, Van Obbergh F, Billiet J, et al. Analysis of phenotype and outcome in essential thrombocythemia with CALR or JAK2 mutations. Haematologica. $\quad$ 2015;100(7):893-897. doi:10.3324/haematol.2014. 118299

20. Malherbe JAJ, Fuller KA, Mirzai B, et al. Dysregulation of the intrinsic apoptotic pathway mediates megakaryocytic hyperplasia in myeloproliferative neoplasms. $J$ Clin Pathol. 2016;69 (11):1017-1024. doi:10.1136/jclinpath-2016-203625

21. Koopmans SM, Schouten HC, van Marion AMW. Anti-apoptotic pathways in bone marrow and megakaryocytes in myeloproliferative neoplasia. Pathobiology. 2014;81(2):60-68. doi:10.1159/000356187

22. Mondet J, Hussein K, Mossuz P. Circulating cytokine levels as markers of inflammation in Philadelphia negative myeloproliferative neoplasms: diagnostic and prognostic interest. Mediators Inflamm. 2015;2015:670580. doi:10.1155/2015/670580

23. Ma C, Fan R, Ahmad H, et al. A clinical microchip for evaluation of single immune cells reveals high functional heterogeneity in phenotypically similar T cells. Nat Med. 2011;17(6):738-743. doi:10.1038/nm.2375

24. Tefferi A, Vaidya R, Caramazza D, et al. Circulating interleukin (IL)-8, IL-2R, IL-12, and IL-15 levels are independently prognostic in primary myelofibrosis: a comprehensive cytokine profiling study. $J \quad$ Clin Oncol. 2011;29(10):1356-1363. doi:10.1200/ JCO.2010.32.9490

25. Pourcelot E, Trocme C, Mondet J, et al. Cytokine profiles in polycythemia vera and essential thrombocythemia patients: clinical implications. Exp Hematol. 2014;42(5):360-368. doi:10.1016/j. exphem.2014.01.006

26. Jones CI, Tucker KL, Sasikumar P, et al. Integrin-linked kinase regulates the rate of platelet activation and is essential for the formation of stable thrombi. J Thromb Haemost. 2014;12(8):1342-1352. doi:10.1111/jth. 12620

27. Tucker KL, Sage T, Stevens JM, et al. A dual role for integrin-linked kinase in platelets: regulating integrin function and $\alpha$-granule secretion. Blood. 2008;112(12):4523-4531. doi:10.1182/blood-2008-03148502

28. Kleppe M, Koche R, Zou L, et al. Dual targeting of oncogenic activation and inflammatory signaling increases therapeutic efficacy in myeloproliferative neoplasms. Cancer Cell. 2018;33(1):29-43.e27. doi:10.1016/j.ccell.2017.11.009

29. Vainchenker W, Constantinescu SN. JAK/STAT signaling in hematological malignancies. Oncogene. 2013;32(21):2601-2613. doi:10.1038/onc.2012.347

30. Kollmann K, Nangalia J, Warsch W, et al. MARIMO cells harbor a CALR mutation but are not dependent on JAK2/STAT5 signaling. Leukemia. 2015;29(2):494-497. doi:10.1038/leu.2014.285

31. Kollmann K, Warsch W, Gonzalez-Arias C, et al. A novel signalling screen demonstrates that CALR mutations activate essential MAPK signalling and facilitate megakaryocyte differentiation. Leukemia. 2017;31(4):934-944. doi:10.1038/leu.2016.280

32. Lau WWY, Hannah R, Green AR, Gottgens B. The JAK-STAT signaling pathway is differentially activated in CALR-positive compared with JAK2V617F-positive ET patients. Blood. 2015;125 (10):1679-1681. doi:10.1182/blood-2014-12-618074 


\section{Publish your work in this journal}

Cancer Management and Research is an international, peer-reviewed open access journal focusing on cancer research and the optimal use of preventative and integrated treatment interventions to achieve improved outcomes, enhanced survival and quality of life for the cancer patient.
The manuscript management system is completely online and includes a very quick and fair peer-review system, which is all easy to use. Visit http://www.dovepress.com/testimonials.php to read real quotes from published authors.

Submit your manuscript here: https://www.dovepress.com/cancer-management-and-research-journal 